\title{
The Role of Transesophageal Echocardiography in Device Closure of Peri-membranous Ventricular Septal Defects with the Hybrid Approach
}

\author{
Manisha Aluri ${ }^{1}$, Fahad Alfares ${ }^{1}$, and Satinder Sandhu ${ }^{1}$ \\ ${ }^{1}$ University of Miami Miller School of Medicine
}

January 28, 2022

\begin{abstract}
Key Points - Perventricular device closure of peri-membranous ventricular septal defects is safe and effective when compared to conventional surgery and transcatheter device closure. - Intraprocedural transesophageal echocardiography can effectively guide perventricular device closure of peri-membranous ventricular septal defects and improve safety and success rate. · Hybrid approach improves the outcomes in select patients with congenital heart diseases and complex anatomical defects.
\end{abstract}

The Role of Transesophageal Echocardiography in Device Closure of Peri-membranous Ventricular Septal Defects with the Hybrid Approach

Manisha Aluri, Fahad Alfares, Satinder K Sandhu

Key Points

- Perventricular device closure of peri-membranous ventricular septal defects is safe and effective when compared to conventional surgery and transcatheter device closure. • Intraprocedural transesophageal echocardiography can effectively guide perventricular device closure of peri-membranous ventricular septal defects and improve safety and success rate. - Hybrid approach improves the outcomes in select patients with congenital heart diseases and complex anatomical defects.

\section{Corresponding Author Information:}

Dr. Satinder Sandhu MD

Professor of Pediatrics

Director, Pediatric Cardiac Cath Laboratory

Director, Adult Congenital Heart Disease

1611 NW 12th Avenue, NW Room109

Miami, Florida, 33136

Phone: 305-585-6683

Fax: 305-324-6012

Email: ssandhu3@med.miami.edu

Acknowledgements : The authors have no acknowledgments to mention 
In 1966 Drs. Rashkind \& Miller set up the stage for transcatheter therapy in congenital heart disease with the publication "Creation of an atrial septal defect without thoracotomy: palliative approach to complete transposition of the great arteries"(1). Since then transcatheter therapy has become the main stay for repair and palliation of select congenital heart defects resulting in improved outcomes. Over the last two decades this has further expanded into hybrid procedures in which the pediatric cardiothoracic surgeons, pediatric interventional cardiologists and pediatric echocardiographers collaborate to perform the procedure with the least invasive approach. An integral part of the success of the hybrid procedures has been the role of transesophageal echocardiography (TEE) in guiding the intervention without the need for fluoroscopy thereby minimizing radiation exposure to the patient $(2,3)$. A meta-analysis of transcatheter device closure of peri-membranous ventricular septal defects (Pm-VSDs) showed a high success rate of device implantation with acceptable margin of safety and rate of complications (4). In patients where transcatheter approach is challenging an alternative approach with comparable results to the transcatheter approach is the role of per ventricular closure with a device through a minimally invasive incision without the need for cardiopulmonary bypass (5).

Yu Jin et al (6) in their report focus on the value of intraprocedural TEE to assist with device closure of the Pm-VSD through the per-ventricular approach. The study is retrospective and includes 207 children with Pm-VSD who underwent device closure of the defect under TEE guidance through a "ultra-minimal trans intercostal incision". The practical ease of the procedure and the benefits of using TEE intra-procedurally were emphasized in this report. TEE was invaluable in selecting the access site on the right ventricle, guiding the positioning of the guidewire and the delivery sheath. The authors concluded that the clarity of images obtained by TEE during this procedure helped in navigating the anatomically difficult lesions, device selection and positioning to prevent interference of the device with the aortic and mitral valve. TEE also assessed the residual shunt and stability of the device prior to and after release. In this report the rate of successful device occlusion of the Pm-VSDs was 97.64\%. Overall this paper has demonstrated that TEE guided closure of a Pm-VSD via minimally invasive device closure is a safe and effective procedure with excellent outcomes when performed by an experienced imager. This has been demonstrated in other studies where TEE was used to guide similar procedures with good outcomes (7).

Per ventricular approach for closure of ventricular septal defects was first described by Amin and colleagues in 1998 (8). This approach should be considered in patients who are too small for transcatheter closure, patients with limited vessel access and in patients with complex VSD anatomy where device placement and device manuverability may be challenging. The advantage of not requiring or shortening the time of cardiopulmonary bypass is also an added value to the procedure. The success of the procedure is dependent on intra-procedural imaging by TEE in order to assess the anatomy of the defect and to mitigate the potential complications of device closure by guiding the device delivery and adjusting or repositioning the device if needed $(9,10)$. The use of TEE during the hybrid approach gives a wider field of view thereby allowing access to the lesions where percutaneous delivery of the device is restricted (11).

Hybrid procedures minimize the perioperative morbidity and improve the long term outcomes in patients. This approach utilizes the strengths and minimizes the limitations of the individual procedures. The future in the treatment of congenital heart disease will be a collaborative effort to get the best outcomes with the least invasive procedure.

\section{References:}

1. Rashkind W J, Miller W, Creation of an atrial septal defect without thoracotomy: palliative approach to complete transposition of the great arteries, JAMA (1966);196: pp. 991-992.

2. Van Der Velde ME, Perry SB. Transesophageal Echocardiography During Interventional Catheterization in Congenital Heart Disease. Echocardiography. 1997 Sep;14(5):513-528

3. Holzer R, de Giovanni J, Walsh KP, Tometzki A, Goh T, Hakim F, Zabal C, de Lezo JS, Cao QL, Hijazi ZM. Transcatheter closure of perimembranous ventricular septal defects using the amplatzer membranous VSD occluder: immediate and midterm results of an international registry. Catheter Cardiovasc Interv. 2006 
Oct;68(4):620-8

4. Santhanam H, Yang L, Chen Z, Tai BC, Rajgor DD, Quek SC. A meta-analysis of transcatheter device closure of perimembranous ventricular septal defect. Int J Cardiol. 2018 Mar 1;254:75-83.

5. Li D, Zhou X, Li M, An Q. Comparisons of perventricular device closure, conventional surgical repair, and transcatheter device closure in patients with perimembranous ventricular septal defects: a network metaanalysis. BMC Surg. 2020 May 26;20(1):115.

6. Yu Jin, Ye Jingjing, Zhang Newei, Yang Xiuzhen, Ma Lianglong, Qian Jinging, Zhao Lei, Qiang Shu: Value of transesophageal echocardiography in device closure of perimembranous ventricular septal defects in children via ultra -minimal trans intercostal incision. In press

7. Ren C, Wu C, Pan Z, Li Y. Minimally invasive closure of transthoracic ventricular septal defect: postoperative complications and risk factors. J Cardiothorac Surg. 2021 Mar 19;16(1):30

8. Amin Z, Berry JM, Foker JE, Rocchini AP, Bass JL. Intraoperative closure of muscular ventricular septal defect in a canine model and application of the technique in a baby. J Thorac Cardiovasc Surg. 1998 Jun;115(6):1374-6

9. Quansheng X, Silin P, Zhongyun Z, Youbao R, Shengde L, Qian C, Shuhua D, Kefeng H, Zhixian J, Qin W. Minimally invasive perventricular device closure of an isolated perimembranous ventricular septal defect with a newly designed delivery system: preliminary experience. J Thorac Cardiovasc Surg. 2009 Mar;137(3):556-9

10. Hu X, Peng B, Zhang Y, Ai F, Zheng J. Short-Term and Mid-Term Results of Minimally Invasive Occlusion of Ventricular Septal Defects via a Subaxillary Approach in a Single Center. Pediatr Cardiol. 2019 Jan;40(1):198-203

11. Michel-Behnke I, Ewert P, Koch A, Bertram H, Emmel M, Fischer G, Gitter R, Kozlik-Feldman R, Motz R, Kitzmuller E, Kretschmar O; Investigators of the Working Group Interventional Cardiology of the German Association of Pediatric Cardiology. Device closure of ventricular septal defects by hybrid procedures: a multicenter retrospective study. Catheter Cardiovasc Interv. 2011 Feb 1;77(2):242-51. 